\title{
Liver-Pancreas-Heart Triangle and HCV in Thalassemia: Expanding the Horizon through Biomarker Networks
}

\author{
Cristina Vassalle*, Antonella Meloni, Laura Pistoia, Alessia Pepe
}

Fondazione G. Monasterio CNR-Regione Toscana, Pisa, Italy

*Corresponding author: Cristina Vassalle, PhD, Fondazione G.Monasterio CNR-Regione Toscana Via Moruzzi 1, I-56124, Pisa, Italy, Tel: +39-050-3153525; Fax: +39-050-3152166, E-mail: cristina.vassalle@ftgm.it

\begin{abstract}
Magnetic Resonance Imaging represents the elective strategy to assess iron overload in different organs and drives the iron chelation therapy in Thalassemia major (TM). However, affected organs, in particular liver, pancreas and heart, are not isolated but interact each other and communicate through biochemical mediators, which may accelerate and worsen pathological processes considered unrelated. A better understanding of these intermingled pathways might help to identify more significant biomarker combinations and the association of these biomarkers to TM complexity, possibly assisting imaging techniques in the prevention of complications and in the improvement of life quality in TM patients.
\end{abstract}

Received Date: January 17, 2017

Accepted Date: February 22, 2017

Published Date: February 27, 2017

Citation: Vassalle, C., et al. Liver-Pancreas-Heart Triangle and HCV in Thalassemia: Expanding the Horizon through Biomarker Networks. (2017) Int J Hematol Ther 3(1): 1- 6 .

DOI: $10.15436 / 2381-1404.17 .017$

Keywords: Liver; Pancreas; Heart; Bone; Thalassemia major; Biomarkers



\section{Introduction}

$\beta$-thalassemia is an inherited autosomal recessive blood disease related to the reduced or absent synthesis of the beta globin chains of hemoglobin ${ }^{[1]}$. The severity of the disease depends on the mutation type: no $\beta$ chains formation in Thalassemia Major (TM), the most severe form characterized by transfusion-dependent type; partial $\beta$ chain formation in thalassemia intermedia, which includes different forms from the asymptomatic carrier state to the severe transfusion-dependent anemia; heterozygosity for $\beta$-thalassemia, essentially asymptomatic in $\beta$-thalassemia carrier status ${ }^{[1]}$.

Apart bone marrow transplantation, management of TM fundamentally consists in regular long-life transfusions and iron chelation therapy (significantly improved with the introduction of the oral chelators deferiprone and deferasirox) to avoid iron overload ${ }^{[1]}$. Evaluation of liver and heart iron with Magnetic Resonance Imaging (MRI) represents a recognized helpful tool to drive the effectiveness of chelation therapy in $\beta$-thalassemia follow-up ${ }^{[2]}$. MRI quantification of pancreatic iron, and evaluation of its relationship with cardiac and liver iron is under de- velopment ${ }^{[3]}$. Moreover, the role of MRI is emerging as more and more imperative as the need of a precise estimate of iron accumulation in different organs increases, because each available iron chelator seems to have different efficacy in each different organ ${ }^{[4]}$. Although the prognosis of thalassemic patients has improved with these pharmacological and instrumental advances, cardiac disease, together with hepatic and endocrinological complications with diabetes mellitus as major endocrinopathy, remain the major sources of mortality and morbidity in such patients and are mainly related to excessive iron accumulation ${ }^{[5,6]}$. Moreover, being high the prevalence of hepatitis $\mathrm{C}$ virus (HCV) infection in these multi-transfused patients, the crucial role of $\mathrm{HCV}$ in the pancreas, liver and cardiac damage through development of diabetes, and myocardial fibrosis (independent from myocardial iron accumulation) is also arising ${ }^{[7,8]}$. In this context, the use of biochemical markers, which represent indices of a biological state or condition, noninvasively measurable in biological samples, might contribute to fill the gap about the liver-heart-pancreas devil's triangle and retain possible additive utility in the clinical practice. 
This review aims to discuss available findings in this research area, illustrating the complexity of relationships between organs, biomarkers and diseases in TM, and the need of a multidisciplinary, holistic approach to improve knowledge of underlying mechanisms which link organs and may potentially assist in the management and decision-making of such patients.

\section{The liver-pancreas-heart triangle, NTproBNP, and diabetes mellitus}

As iron accumulates primarily in hepatocytes, liver damage may be related to development of fibrosis and cirrhosis and, potentially, to hepatocellular cancer ${ }^{[1]}$. Moreover, iron-induced injury early extends to the pancreas (decreased insulin secretion and diabetes), endocrine system (hypogonadism, thyroid dysfunction, etc), and heart (cardiomiopathy and arrhythmias) [1]. The range of abnormalities demonstrates the complex pathophysiology of TM disease ${ }^{[5]}$. The situation is more complicated by the observation that organs are closely interrelated through production of mediators, and disease of one organ may cause dysfunction of others and vice versa, ultimately contributing to aggravate general failure.

In particular, the liver-pancreas-heart triangle emerges as central in the network of organs that regulate energy and metabolism, and different biochemical markers produced by these organs emerge as key mediators in the metabolic dysregulation ${ }^{[9]}$. In this context, the onset of insulin resistance and decreased insulin secretion are both recognized as pivotal factors for diabetes risk in TM patients ${ }^{[5]}$. The increase in hepatic iron induces reactive oxygen species (ROS) and initiates a pro-inflammatory response, activating NF- $\mathrm{KB}$ intracellular signaling cascades, increasing IL- 6 , TNF- $\alpha$, and IL- $1 \beta$, which drive hepatic insulin resistance ${ }^{[5,9]}$. Iron begins to accumulate in pancreas in early childhood, and the prevalence of diabetes mellitus (DM) is significant and may reach $14 \%$ in TM patients ${ }^{[10]}$. Impaired Beta-cell function, as measured by a reduction in the insulin secretion index, characterizes $\beta$-thalassaemic patients with normoglycaemia, even before evident alterations in oral glucose tolerance tests.

The criteria defining diabetes risk in thalassemic patients should be clearly different respect to general population. Interestingly, in a study on thalassemic patients $(\mathrm{n}=59)$, the attempt based on ROC analysis to define specific cut-off for glucose and insulin in the thalassemic population, evidenced that the value of $97 \mathrm{mg} / \mathrm{dL}$ for fasting glucose (instead of $100 \mathrm{mg} / \mathrm{dL}$ normally considered), and $9 \mathrm{uU} / \mathrm{mL}$ (instead of $20 \mathrm{uU} / \mathrm{mL}$ ) for fasting insulin accurately identified an abnormal result for oral glucose test tolerance (OGTT) ${ }^{[11]}$. The authors proposed an annual screening of fasting glucose/insulin measurement coupled with MRI evaluation of iron pancreas (pancreas R2*) as tool to identify patients at high risk of glucose dysregulation ${ }^{[11]}$. Pancreatic beta cell function (Dispositon index) resulted to be correlated with pancreas iron (R2*) while insulin resistance resulted associated with serum ferritin, and liver iron concentration ${ }^{[11]}$. Cardiac R2* appeared more specific to identify glucose dysfunction, which implies pancreatic iron accumulation for a long duration, but less sensitive to milder glucose dysregulation ${ }^{[11]}$. In this study, no association was observed between glucose alteration and levels of other biochemical markers, as vitamin D $25 \mathrm{OH}$, intact parathyroid hormone or zinc ${ }^{[11]}$.

Other data indicate that DM in thalassemia correlates with ferritin concentration, hepatitis infection, and pancreatic and cardiac iron measured by imaging techniques ${ }^{[10]}$. At present, no study has investigated the role of pro-brain natriuretic peptide (NT-proBNP), produced in the heart and well known for its central role in the $\mathrm{CV}$ system, in such relationship. In $\beta$-thalassemic patients, high NT-proBNP significantly correlates with documented left ventricular diastolic dysfunction, and retains predictive value in latent left ventricular diastolic dysfunction ${ }^{[12]}$. Moreover, there is a significant association between NT-proBNP levels and cardiac iron concentration in TM patients, evidencing the relationship between biochemical and imaging techniques ${ }^{[13]}$. New unexpected effects of natriuretic peptides (NP) have been recently evidenced, which suggest a role for these biomarkers as metabolic hormones. In particular, clinical studies have shown that although increased NP levels characterize cardiac dysfunction and heart failure, reduced natriuretic peptides levels are associated with metabolic diseases such as obesity and type 2 diabetes T2 $\mathrm{D}^{[14]}$. Moreover, a low NP level also predicts the risk of new onset $\mathrm{T} 2 \mathrm{D}^{[15]}$. Experimental studies have also confirmed a key role of NP in the regulation of energy balance and glucose homeostasis ${ }^{[16]}$. Regardless underlying mechanisms, they remain largely unknown, although some data suggest that the brain natriuretic peptide (BNP) would play a direct action on muscle and adipose tissue, probably involving adiponectin secretion and insulin resistance ${ }^{[14]}$

Interestingly, a common genetic BNP polymorphism (rs198389), which results in a $20 \%$ increase in NTproBNP levels, was associated with a $15 \%$ reduced risk of T2D $\mathrm{D}^{[17]}$. However, there are no data exploring the association between NTproBNP, glucose metabolism and diabetes in thalassemic cohorts.

Oxidative stress and inflammation drive insulin resistance and may account for the higher DM risk in TM. At now, the role of NTproBNP in the onset and development of diabetes and its relationship with other biomarkers related to metabolic control has not been evaluated at all in TM, and merits attention in future studies.

\section{Iron homeostasis, hepcidin, and glucose metabolism}

Iron, which is increasingly recognized to influence glucose metabolism at multiple levels, has been related to worsened insulin sensitivity and to the pathogenesis of $\mathrm{DM}^{[18]}$. Body iron metabolism is characterized by a complex network of storage and transport which is regulated by several factors ${ }^{[19]}$. Nonetheless the protein hepcidin, secreted mainly by the liver, is emerging as a major iron regulatory factor ${ }^{[19]}$. Hepcidin regulates iron absorption in the intestine and its release from macrophages. The lack of hepcidin increases duodenal iron absorption, and results in iron overload ${ }^{[19]}$. Erythropoiesis, anemia and hypoxia down-regulate hepcidin, whereas hepcidin is elevated by inflammation and iron overload ${ }^{[19]}$. Interestingly, recent data in TM patients showed that pre- and post-transfusion hepcidin values were positively associated with hemoglobin and ferritin, and inversely with erythropoiesis, presenting gender-differences as male patients showed pre- and post-transfusional increased erythropoiesis and lower hepcidin as compared with female patients ${ }^{[20]}$. Authors proposed the evaluation of hepcidin (together with the hepcidin-ferritin ratio) as biomarker of erythropoietic and iron-loading signals. However, recent data evidenced comparable levels of serum ferritin, serum hepcidin, and hepcidin/ ferritin ratio in patients $(n=100)$ with or without detectable MRI 
cardiac iron (T2* cut off corresponding to $20 \mathrm{~ms})^{[21]}$. The potential of hepcidin as possible additive tool in the management of thalassemia is still under study, and all these data clearly need further confirmation, but are very interesting also in view of recent findings evidencing a role for hepcidin in the pathophysiology of insulin resistance and T2D, a frequent complication in thalassemic patients. Mechanisms underlying this relationship are still under investigation, although likely involve interrelated pathway of STAT3 (transcription modulator of hepcidin), pro-inflammatory cytokines (IL-6, in particular, also including IL-1, TNF- $\alpha$ and IL-10), and the TMPRSS6 enzyme (inhibitor of hepcidin expression) ${ }^{[22]}$. Specially, IL-6 represents the most critical cytokine regulating hepcidin, and has a recognized role in TD2 pathogenesis $^{[23]}$. It is supposed that IL-6 may act through STAT3 phosphorylation, activating STAT3 and hepcidin expression ${ }^{[24]}$.

The relationship between iron metabolism and insulin resistance is recognized bidirectional ${ }^{[25]}$. Whether insulin is known to increase ferritin synthesis and iron uptake, iron-induced damage drives alterations of glucose metabolism through enhanced oxidative stress and inflammation ${ }^{[25]}$.

In this context, it is also plausible that low hepcidin bioavailability could stimulate IL-6, thereby enhancing its role in the development of insulin resistance and T2D.

The importance of hepcidin as a final common mediator at the crossroad of systemic and cellular iron metabolism, inflammation, oxidative stress, erythropoiesis, and hypoxia is emerging. Moreover, the relationship of hepcidin with cytokines, STAT3 and oxidative stress makes this molecule a candidate in the pathogenesis of diabetes.

Now, a better knowledge of hepcidin alterations and its relationship with biochemical and imaging markers may expand our understanding of underlying mechanisms and risk factors involved in TM and its metabolic complications, as well as to evaluate the therapeutic potential of approaches to modulate hepcidin levels in thalassemic patients.

\section{Oxidative stress and inflammatory markers as index of ath- erosclerosis in TM}

When the iron binding capacity of transferrin is saturated, circulating iron appear in a free form, non-transferrin-bound iron (NTBI), a powerful inducer of free radicals ${ }^{[26]}$. Excess iron increases oxidative stress, which in turn impairs cellular function and integrity, causing damage to mitochondria, lysosomes, lipid membranes, proteins, and DNA ${ }^{[27]}$.

Interestingly, with the transfusions and chelating agent advancement, the life expectance in TM patients has increased significantly and, from pediatric and adolescence pathology, changing to become an adulthood and elderly disease in future. Thus, the role of classically age-related pathological conditions begin to have significance also in TM. In particular, it is known that oxidative stress has a pivotal role of in the onset and development of atherosclerosis ${ }^{[28]}$.

Different findings suggest that iron-derived free-radicals may cause premature, early subclinical signs of atherosclerosis (increased carotid arterial thickness and stiffness) in thalassemia patients. A more recent study ( $31 \mathrm{TM}$ children, 36 healthy children; age 4 - 16 years) considered the additive dimension of laboratory parameters, evidencing a relationship between development of premature atherosclerosis by carotid intima-media thickness and a biochemical pro-atherogenic profile ${ }^{[29]}$. To- tal cholesterol, low-density lipoprotein (LDL) cholesterol and high-density lipoprotein (HDL) cholesterol levels resulted significantly reduced, whereas very-low-density lipoprotein cholesterol levels were significantly elevated in $\beta$-thalassemic patients compared to control subjects ${ }^{[29]}$. Moreover, nitrate/nitrite (NOx), as index of endothelial function, was significantly reduced, instead chitotroisidase enzyme activity (an index of macrophage activation) as well as carotid intima-media thickness (IMT) were significantly increased in the TM patient group compared to healthy subjects, and NOx significantly correlated with carotid $\mathrm{IMT}^{[29]}$. These data were in agreement with previous ones, where $\beta$-thalassemia patients were characterized by a predominance of small-dense LDL particles, increased oxidative stress (8-isoprostane), and Lipoprotein-associated phospholipase A(2) (LpPLA(2), inflammatory enzyme implicated in atherosclerosis) mass and activity ${ }^{[30]}$. A significant positive correlation between plasma Lp-PLA(2) activity or mass and 8-isoprostane and ferritin levels as well as with IMT was also observed ${ }^{[30]}$. An increase in secreted and cell-associated Lp-PLA(2) activity was also observed in culture of monocytes from $\beta$-thalassemic patients ${ }^{[30]}$. Moreover, tumor necrosis factor- $\alpha$ (TNF- $\alpha$, another inflammatory biomarker) and Lp-PLA2 levels resulted high in thalassemia children and both biomarkers showed strong correlations with IMT $^{[31]}$.

Other data showed that total antioxidant capacity (TAC) and Paraoxonase-1 (PON1, with protective property against lipid oxidation) resulted decreased, while malondialdehyde (MDA) increased in $\beta$-thalassaemia subjects ${ }^{[32]}$. Decrease in PON1 activity resulted associated with the degree of oxidative stress, anaemia and increase in IMT $^{[32]}$. Other authors have evidenced that PON1 activity was significantly lower in TM children than controls, whereas total peroxide concentration levels resulted significantly higher ${ }^{[33]}$.

Indices of vessel dysfunction, as Willebrand factor antigen (VWF:Ag) and flow cytometric analysis of circulating CD144(+) endothelial microparticles (EMPs) and endothelial progenitor cells $[\mathrm{CD} 34(+) \mathrm{VEGFR} 2(+)]$, resulted significantly higher among patients with $\beta$-thalassemia than controls ${ }^{[34]}$. Also D-dimer, and other circulating markers of endothelial activation, inflammation and apoptosis resulted higher, while plasma cholesterol and fibrinogen levels were lower than normal in TM patient $^{[35]}$.

Very recently, some polymorphisms related to biomarkers of oxidative stress and endothelial function have been identified as possible genetic markers for prediction of increased susceptibility to cardiovascular complications in TM. In particular, Hp2-2 polymorphism of Haptoglobin (Hp), an antioxidant protein, which polymorphism has been related to vascular complications among diabetic TM patients, resulted a significant predictor for premature atherosclerosis (IMT) in TM children ${ }^{[36]}$. Moreover, the frequency of eNOS4a allele (aa and ab genotypes), polymorphism of the endothelial nitric oxide synthase (eNOS, enzyme that generates nitric oxide major determinant of endothelial function), was significantly higher in $\beta$-TM patients with pulmonary hypertension or cardiomyopathy, and could represent a possible genetic marker for prediction of increased susceptibility to cardiovascular complications in $\mathrm{TM}^{[37]}$.

TM subjects may be more prone to development of atherosclerosis. A biochemical proatherogenic profile has been evidenced in TM, and there is a correlation between different 
biochemical and genetic markers and subclinical indices of atherosclerosis. However, most biomarkers were investigated in a single cohort study or population and need to be replicated in more studies. There are no data which evaluated the correlation between a pro-atherogenic biochemical profile and MRI iron accumulation in heart and other organs. At present, if these biomarkers could represent possible indices of $\mathrm{CV}$ complications in $\mathrm{TM}$ remains to be determined.

\section{HCV and the liver-pancreas-heart triangle}

Transfusion-acquired HCV infection is common in ${ }^{T M}{ }^{[38]}$. HCV infection may represent a strong accelerator of TM complications, as elicits chronic inflammation (especially IL- 6 and TNF- $\beta$ ) and oxidative stress, induces insulin resistance, and increases the risk of diabetes and metabolic syndrome and liver damage ${ }^{[39]}$. The mechanisms of HCV complications development also include immunological effects, as the chronic persistence of virus leads to the circulation of immune complexes (mixed cryoglobulinemia) and other autoimmune response ${ }^{[40]}$. Some data suggest that viral proteins such as HCV-core, E1, and NS3 can impair mitochondrial respiratory chain enzymes and different cellular pathways, and expression of HCV proteins (e.g. NS5A) increases oxidative stress interfering with several pathways, including the activation of STAT-3 and Nuclear factor $\kappa \mathrm{B}(\mathrm{NF}-\kappa \mathrm{B})$ and calcium signaling ${ }^{[41]}$.

It is known that patients with chronic $\mathrm{HCV}$ have a high prevalence of glycometabolic abnormalities, from insulin resistance to diabetes ${ }^{[42]}$. Interestingly, recent data evidenced gender-related differences for metabolic risk in HCV patients. Specifically, women resulted at increased risk for insulin resistance (IR) in HCV, and also an increased risk of postmenopausal metabolic syndrome was found ${ }^{[43]}$. IR is also associated with increased liver fibrosis in chronic HCV patients ${ }^{[4]}$. Recent data indicate that HCV-NS5A is implicated in the induction of insulin resistance by modulating various cellular targets involved in the insulin signaling pathway, in particular through phosphorylation of IRS-1 serine and increased gluconeogenesis ${ }^{[45]}$. Moreover, HCV core protein induces alterations in cellular redox state $(\mathrm{NAD}(+) / \mathrm{NADH}$ reduction), and, likely through the SIRT1/ AMPK pathway, with a key role in the regulation of hepatic glucose and lipid metabolism modifies the expression profile of glucose and lipid metabolism-related genes, causing metabolism dysregulation in HepG2 cells ${ }^{[46]}$. The simple neutrophil/ lymphocyte ratio, an old index which integrates information on the inflammatory and physiological stress, resulted higher in patients with chronic HCV, HOMA-IR > 3 and advanced fibrosis, promising as possible noninvasive parameter to predict IR and advanced fibrosis stages in chronic $\mathrm{HCV}$, and could be useful also in TM patients ${ }^{[47]}$.

Interestingly, YKL-40, an inflammatory glycoprotein, that resulted elevated in patients with CVD or HCV, is also correlated with liver stiffness and the degree of hepatic fibrosis in TM patients with liver disease ${ }^{[48]}$. YKL-40 also resulted positively correlated with transfusion index, alanine aminotransferase, lactate dehydrogenase, serum ferritin and liver iron concentrations (LIC), and negatively correlated with cardiac MRI-T2*, data which render YKL-40 attractive as additive tool to estimate liver fibrosis in $\mathrm{TM}^{[48]}$.

In the last years, many studies have evaluated the relationship between HCV and atherosclerosis ${ }^{[49-51]}$. However many findings evidenced a close correlation between carotid or coronary atherosclerosis and $\mathrm{HCV}$, some other results did not confirm such association ${ }^{[49]}$. Interestingly, an higher mortality for $\mathrm{CV}$ cause was observed in anti-HCV positive patients ${ }^{[52]}$. Moreover, HCV RNA values were associated with excess cerebrovascular mortality in a large general population (23 820 adults aged 30-65 years old, follow-up 1991-2008) $)^{[33]}$.

An aspect against the pro-atherogenic role of $\mathrm{HCV}$ is the finding of hypocholesterolemia, which characterized HCV patients $^{[54]}$. Clearly, alteration in lipoprotein and apolipoprotein may be secondary to worsened liver function induced by $\mathrm{HCV}$, although HCV infection itself might directly cause hypolipidemia $^{[55]}$. Accordingly, recent data suggest that the disturbance of the lipoprotein metabolism, as increase of LDL-TG and small VLDL-TG (highly atherogenic), is an independent factor associated with chronic active HCV G1b infection with advanced fibrosis $^{[56]}$. This result indirectly suggests that a high level of small VLDL-TG might be one of the risk factors for atherosclerosis in $\mathrm{HCV}$ infection with advanced fibrosis, although the role of VLDL-TG and atherosclerosis remains to be determined. Moreover, abnormalities in HDL and apolipoprotein levels in HCV are emerging, although significance of these anomalies in terms of atherosclerosis effects, and the differences related to different $\mathrm{HCV}$ genotypes, have not been clarified ${ }^{[57]}$.

Chronic HCV is often associated with altered iron homeostasis, and some data suggest that it suppresses hepcidin expression, worsening iron overload, likely through mechanism which involve oxidative stress, inflammation, and the IL-6/ STAT3 pathway ${ }^{[58]}$.

An altered lipid metabolism associated with oxidative stress, inflammation and liver fibrosis characterized chronic HCV infection. Furthermore, same underlying mechanisms may likely favor atherosclerosis and worsened iron overload in chronic HCV. Further studies are required to understand the effects of these interactions in TM.

\section{Conclusion}

Patients with TM display interrelated changes in systemic biomarkers, such as indices of inflammation, oxidative stress, and endothelial function, and a number of other biomarkers at the level of different organs. Raising data suggest the role for bone in the regulation of metabolism, in the pathogenesis of atherosclerotic processes, and liver fibrosis, with relationships between organs that are increasingly emerging as mutual and reciprocal ${ }^{[59,60]}$. Although likely MRI will continue to dominate the clinical practice of TM, identification of more significant biomarkers in terms of prognosis and better elucidation of network functioning could give a deeper understanding of the complex molecular network underlying TM, which, in turn, could potentially pave the way to the development of additive multiple-purpose complication preventive measures and therapeutic tools.

\section{Acknowledgment}

The authors declare that there is no conflict of interest regarding the publication of this paper. 


\section{References}

1. Cao, A., Galanello, R. Beta-thalassemia. (2010) Genet Med 12(2): 61-76.

Pubmed I Crossref

2. Pepe, A., Meloni, A., Rossi, G., et al. Cardiac and hepatic iron and ejection fraction in thalassemia major: multicentre prospective comparison of combined deferiprone and deferoxamine therapy against deferiprone or deferoxamine monotherapy. (2013) J Cardiovasc Magn Reson 15:1.

Pubmed I Crossref

3. Meloni, A., Restaino, G., Missere, M., et al. Pancreatic iron overload by T2* MRI in a large cohort of well treated thalassemia major patients: Can it tell us heart iron distribution and function? (2015) Am J Hematol 90(9): E189-190.

Pubmed I Crossref

4. Pepe, A., Meloni, A., Capra, M., et al. Deferasirox, deferiprone and desferrioxamine treatment in thalassemia major patients: cardiac iron and function comparison determined by quantitative magnetic resonance imaging. (2011) Haematologica 96(1): 41-47.

Pubmed I Crossref

5. De Sanctis, V., Soliman, A.T., Elsedfy, H., et al. Diabetes and Glucose Metabolism in Thalassemia Major: An Update. (2015) Expert Review of Hematology 9(4): 401-408.

Pubmed I Crossref

6. Borgna-Pignatti, C., Rugolotto, S., De Stefano, P., et al. Survival and complications in patients with thalassemia major treated with transfusion and deferoxamine. (2004) Haematologica 89(10): 1187-1193.

Pubmed

7. Pepe, A., Meloni, A., Borsellino, Z., et al. Myocardial fibrosis by late gadolinium enhancement cardiac magnetic resonance and hepatitis $\mathrm{C}$ virus infection in thalassemia major patients. (2015) J Cardiovasc Med (Hagerstown) 16(10): 689-695.

Pubmed I Crossref

8. Pepe, A., Positano, V., Capra, M., et al. Prevalence and clinical-Instrumental correlates of myocardial scarring by delayed enhancement cardiovascular magnetic resonance in thalassemia major. (2009) Heart 95: 1688-1693.

Crossref

9. Nolan, C.J., Damm, P., Prentki, M. Type 2 diabetes across generations: from pathophysiology to prevention and management. (2011) Lancet 378(9786): 169-181.

Pubmed I Crossref

10. De Sanctis, V., Soliman, A., Yassin, M. Iron overload and glucose metabolism in subjects with beta-thalassaemia major: an overview. (2013) Curr Diabetes Rev 9(4): 332-341.

Pubmed I Crossref

11. Noetzli, L.J., Mittelman, S.D., Watanabe, R.M., et al. Pancreatic iron and glucose dysregulation in thalassemia major. (2012) Am J Hematol 87(2): 155-160.

Pubmed I Crossref

12. Kremastinos, D.T., Hamodraka, E., Parissis, J., et al. Predictive value of B-type natriuretic peptides in detecting latent left ventricular diastolic dysfunction in beta-thalassemia major. (2010) Am Heart J 159(1): 68-74.

Pubmed I Crossref

13. Delaporta, P., Kattamis, A., Apostolakou, F., et al. Correlation of NT-proBNP levels and cardiac iron concentration in patients with transfusion-dependent thalassemia major. (2013) Blood Cells Mol Dis 50(1): 20-24.

Pubmed I Crossref

14. Coue, M., Moro, C. Natriuretic peptide control of energy balance and glucose homeostasis. (2016) Biochimie 124: 84-91.

Pubmed I Crossref

15. Magnusson, M., Jujic, A., Hedblad, B., et al. Low plasma level of atrial natriuretic peptide predicts development of diabetes: the prospective Malmo Diet and Cancer study. (2012) J Clin Endocrinol Metab
97(2): 638-645.

Pubmed I Crossref

16. Plante, E., Menaouar, A., Danalache, B.A., et al. Treatment with brain natriuretic peptide prevents the development of cardiac dysfunction in obese diabetic db/db mice. (2014) Diabetologia 57(6): 12571267.

Pubmed I Crossref

17. Pfister, R., Sharp, S., Luben, R., et al. Mendelian randomization study of B-type natriuretic peptide and type 2 diabetes: evidence of causal association from population studies. (2011) PLoS Med 8(10): e1001112.

Pubmed I Crossref

18. Fernandez-Real, J.M., McClain, D., Manco, M. Mechanisms Linking Glucose Homeostasis and Iron Metabolism Toward the Onset and Progression of Type 2 Diabetes. (2015) Diabetes Care 38(11): 21692176.

Pubmed I Crossref

19. Casu, C., Rivella, S. Iron age: novel targets for iron overload. (2014) Hematology Am Soc Hematol Educ Program 2014(1): 216-221.

Pubmed I Crossref

20. Pasricha, S.R., Frazer, D.M., Bowden, D.K., et al. Transfusion suppresses erythropoiesis and increases hepcidin in adult patients with beta-thalassemia major: a longitudinal study. (2013) Blood 122(1): 124133.

Pubmed I Crossref

21. Said Othman, K.M., Elshazly, S.A., Heiba, N.M. Role of non-invasive assessment in prediction of preclinical cardiac affection in multi-transfused thalassaemia major patients. (2014) Hematology 19(7): 380-387.

Pubmed I Crossref

22. Aregbesola, A., Voutilainen, S., Virtanen, J.K., et al. Serum hepcidin concentrations and type 2 diabetes. (2015) World J Diabetes 6(7): 978-982.

Pubmed I Crossref

23. Jiang, F., Sun, Z.Z., Tang, Y.T., et al. Hepcidin expression and iron parameters change in Type 2 diabetic patients. (2011) Diabetes Res Clin Pract 93(1): 43-48.

Pubmed I Crossref

24. Mayeur, C., Lohmeyer, L.K., Leyton, P., et al. The type I BMP receptor Alk3 is required for the induction of hepatic hepcidin gene expression by interleukin-6. (2014) Blood 123(14): 2261-2268.

Pubmed I Crossref

25. Fernandez-Real, J.M., Lopez-Bermejo, A., Ricart, W. Cross-talk between iron metabolism and diabetes. (2002) Diabetes 51(8): 23482354.

Pubmed I Crossref

26. Brissot, P., Ropert, M., Le Lan, C., et al. Non-transferrin bound iron: a key role in iron overload and iron toxicity. (2012) Biochim Biophys Acta 1820(3): 403-410.

27. Pingitore, A., Lima, G.P., Mastorci, F., et al. Exercise and oxidative stress: potential effects of antioxidant dietary strategies in sports. (2015) Nutrition 31(7-8): 916-922.

Pubmed I Crossref

28. Vassalle, C., Bianchi, S., Battaglia, D., et al. Elevated levels of oxidative stress as a prognostic predictor of major adverse cardiovascular events in patients with coronary artery disease. (2012) J Atheroscler Thromb 19(8): 712-717.

Crossref

29. Gursel, O., Kurekci, A.E., Tascilar, E., et al. Premature atherosclerosis in children with beta-thalassemia major. (2012) J Pediatr Hematol Oncol 34(8): 630-634.

Pubmed I Crossref

30. Tselepis, A.D., Hahalis, G., Tellis, C.C., et al. Plasma levels of lipoprotein-associated phospholipase A(2) are increased in patients with beta-thalassemia. (2010) J Lipid Res 51(11): 3331-3341.

Pubmed I Crossref 
31. Ragab, S.M., Safan, M.A., Obeid, O.M., et al. Lipoprotein-associated phospholipase A2 (Lp-PLA2) and tumor necrosis factor-alpha (TNF-alpha) and their relation to premature atherosclerosis in beta-thalassemia children. (2015) Hematology 20(4): 228-238.

Pubmed I Crossref

32. Labib, H.A., Etewa, R.L., Gaber, O.A., et al. Paraoxonase-1 and oxidative status in common Mediterranean beta-thalassaemia mutations trait, and their relations to atherosclerosis. (2011) J Clin Pathol 64(5): 437-442.

Pubmed I Crossref

33. Cakmak, A., Soker, M., Koc, A., et al. Paraoxonase and arylesterase activity with oxidative status in children with thalassemia major. (2009) J Pediatr Hematol Oncol 31(8): 583-587.

Pubmed I Crossref

34. Adly, A.A., El-Sherif, N.H., Ismail, E.A., et al. Vascular dysfunction in patients with young beta-thalassemia: relation to cardiovascular complications and subclinical atherosclerosis. (2015) Clin Appl Thromb Hemost 21(8): 733-744.

Pubmed I Crossref

35. Hahalis, G., Kremastinos, D.T., Terzis, G., et al. Global vasomotor dysfunction and accelerated vascular aging in beta-thalassemia major. (2008) Atherosclerosis 198(2): 448-457.

Pubmed I Crossref

36. Ragab, S.M., Safan, M.A., Badr, E.A., et al. Haptoglobin genotypes polymorphism as a risk factor for subclinical atherosclerosis in beta-thalassemia major children; a single center Egyptian study. (2015) Hematology 20(8): 477-486.

Pubmed I Crossref

37. Tantawy, A.A., Adly, A.A., Ismail, E.A., et al. Endothelial nitric oxide synthase gene intron 4 variable number tandem repeat polymorphism in beta-thalassemia major: relation to cardiovascular complications. (2015) Blood Coagul Fibrinolysis 26(4): 419-425.

Pubmed I Crossref

38. Pepe, A., Meloni, A., Rossi, G., et al. Cardiac complications and diabetes in thalassaemia major: a large historical multicentre study. (2013) Br J Haematol 163(4): 520-527.

Pubmed I Crossref

39. Pothineni, N.V., Delongchamp, R., Vallurupalli, S., et al. Impact of hepatitis $\mathrm{C}$ seropositivity on the risk of coronary heart disease events. (2014) Am J Cardiol 114(12): 1841-1845.

Pubmed I Crossref

40. Gill, K., Ghazinian, H., Manch, R., et al. Hepatitis C virus as a systemic disease: reaching beyond the liver. (2016) Hepatol Int 10(3): 415-423.

Pubmed I Crossref

41. Lozano-Sepulveda, S.A., Bryan-Marrugo, O.L., Cordova-Fletes, C., et al. Oxidative stress modulation in hepatitis $\mathrm{C}$ virus infected cells. (2015) World J Hepatol 7(29): 2880-2889.

Pubmed I Crossref

42. Modaresi Esfeh, J., Ansari-Gilani, K. Steatosis and hepatitis C. (2016) Gastroenterol Rep (Oxf) 4(1): 24-29.

Pubmed I Crossref

43. Gonzales, C.A., Bacchetti, P., Khalili, M. Impact of gender and menopausal status on metabolic parameters in chronic hepatitis $\mathrm{C}$ infection. (2015) J Viral Hepat 23(3): 232-239.

Pubmed I Crossref

44. Patel, S., Jinjuvadia, R., Patel, R., et al. Insulin Resistance is Associated With Significant Liver Fibrosis in Chronic Hepatitis C Patients: A Systemic Review and Meta-Analysis. (2016) J Clin Gastroenterol 50(1): 80-84.

Pubmed I Crossref

45. Parvaiz, F., Manzoor, S., Iqbal, J., et al. Hepatitis C virus NS5A promotes insulin resistance through IRS-1 serine phosphorylation and increased gluconeogenesis. (2015) World J Gastroenterol 21(43): 12361-12369.

Pubmed I Crossref
46. Yu, J.W., Sun, L.J., Liu, W., et al. Hepatitis $\mathrm{C}$ virus core protein induces hepatic metabolism disorders through down-regulation of the SIRT1-AMPK signaling pathway. (2013) Int J Infect Dis 17(7): e539545.

Pubmed I Crossref

47. Abdel-Razik, A., Mousa, N., Besheer, T.A., et al. Neutrophil to lymphocyte ratio as a reliable marker to predict insulin resistance and fibrosis stage in chronic hepatitis $C$ virus infection. (2015) Acta Gastroenterol Belg 78(4): 386-392.

Pubmed

48. El-Asrar, M.A., Elbarbary, N.S., Ismail, E.A., et al. Serum YKL-40 in young patients with beta-thalassemia major: Relation to hepatitis $\mathrm{C}$ virus infection, liver stiffness by transient elastography and cardiovascular complications. (2016) Blood Cells Mol Dis 56(1): 1-8.

Pubmed I Crossref

49. Adinolfi, L.E., Zampino, R., Restivo, L., et al. Chronic hepatitis $\mathrm{C}$ virus infection and atherosclerosis: clinical impact and mechanisms. (2014) World J Gastroenterol 20(13): 3410-3417.

Pubmed I Crossref

50. Vassalle, C., Masini, S., Bianchi, F., et al. Evidence for association between hepatitis $\mathrm{C}$ virus seropositivity and coronary artery disease. (2004) Heart 90(5): 565-566.

Pubmed I Crossref

51. Vassalle, C. Hepatitis $\mathrm{C}$ virus and atherosclerosis in a close and dangerous liaison. (2012) Atherosclerosis 221(2): 319-320.

Pubmed I Crossref

52. Negro, F., Forton, D., Craxi, A., et al. Extrahepatic morbidity and mortality of chronic hepatitis C. (2015) Gastroenterology 149(6): 1345 1360

Pubmed I Crossref

53. Lee, M.H., Yang, H.I., Lu, S.N., et al. Chronic hepatitis C virus infection increases mortality from hepatic and extrahepatic diseases: a community-based long-term prospective study. (2012) J Infect Dis 206(4): 469-477

Pubmed I Crossref

54. Dai, C.Y., Yeh, M.L., Huang, C.F., et al. Chronic hepatitis C infection is associated with insulin resistance and lipid profiles. (2015) $\mathrm{J}$ Gastroenterol Hepatol 30(5): 879-884.

Pubmed I Crossref

55. Miyazaki, T., Honda, A., Ikegami, T., et al. Hepatitis C virus infection causes hypolipidemia regardless of hepatic damage or nutritional state: An epidemiological survey of a large Japanese cohort. (2011) Hepatol Res 41(6): 530-541.

Pubmed I Crossref

56. Nagano, T., Seki, N., Tomita, Y., et al. Impact of Chronic Hepatitis C Virus Genotype 1b Infection on Triglyceride Concentration in Serum Lipoprotein Fractions. (2015) Int J Mol Sci 16(9): 20576-20594.

Pubmed I Crossref

57. Aizawa, Y., Seki, N., Nagano, T., et al. Chronic hepatitis C virus infection and lipoprotein metabolism. (2015) World J Gastroenterol 21(36): 10299-10313.

Pubmed I Crossref

58. Hino, K., Nishina, S., Hara, Y. Iron metabolic disorder in chronic hepatitis C: mechanisms and relevance to hepatocarcinogenesis. (2013) J Gastroenterol Hepatol 28(Suppl 4): 93-98.

Pubmed I Crossref

59. Raszeja-Wyszomirska, J., Miazgowski, T. Osteoporosis in primary biliary cirrhosis of the liver. (2014) Prz Gastroenterol 9(2): 82-87.

Pubmed I Crossref

60. Vassalle, C., Iervasi, G. New insights for matrix Gla protein, vascular calcification and cardiovascular risk and outcome. (2014) Atherosclerosis 235(1): 236-238.

Pubmed I Crossref 